\title{
Six Exercises from the "111 Training Module" in the Abstract Art Language Course for Painting Majors in Chinese Universities
}

\author{
Dong Shuai ${ }^{1 *}$ Karthiyaini Devarajoo ${ }^{2}$ \\ 1.Qingdao Film Academy, No. 689, Jin sha tan Road, Economic and Technological Development Zone, \\ Qingdao City, Shandong Province \\ 2.Infrastructure University Kuala Lumpur,Unipark Suria, Jalan Ikram-Uniten,43000 Kajang, Selangor Darul \\ Ehsan,Malaysia
}

\begin{abstract}
In 2021, China will enter the "14th Five-Year Plan" period, and the Ministry of Education of China has put forward new requirements for higher education.The Ministry of Education requires the promotion of the integration of vocational education and general education during the "14th Five-Year Plan" period to meet the diverse needs of high-level talents for economic and social development. With the development of the country, the abstract painting courses of painting majors in Chinese colleges and universities should also make corresponding reforms to meet the requirements of the Chinese Ministry of Education.The scope of professional definition of this research is the professional number 130402, painting major, of the Undergraduate Professional Catalogue of Ordinary Colleges and Universities issued by the Ministry of Education of China in 2021.Painting majors include oil paintings, murals, Chinese paintings, prints and many other types of paintings. The title of the course in this study comes from the Decision of the Ministry of Education on Approving the 2018 National Teaching Achievement Award Projects issued by the text number: Teacher [2018] No. 21. In accordance with the provisions of the General University Undergraduate Major Catalog and Major Introduction compiled by the Higher Education Department of the Ministry of Education of the People's Republic of China, the abstract art language course is one of the core courses of the painting major.This research introduces the 6 classroom practice designs of 111 modules in the New Chinese Calligraphy Teaching Method I created. It mainly includes the guiding method of teaching materials and the arrangement sequence of classroom exercises and schematic analysis.

Keywords: abstract painting teaching, new Chinese calligraphy teaching method, abstract sketching module, 111 training module, teaching design

DOI: $10.7176 / \mathrm{JEP} / 13-2-04$

Publication date: January $31^{\text {st }} 2022$

\section{Introduction}

Kandinsky is the inventor of Western abstract painting. Kandinsky's teaching method is derived from Western teaching theories. When Kandinsky invented abstract painting theory, he did not consider the acceptance of the Chinese people (Luo, 2013)Many examples in his teaching methods involve Western cultural habits and Western classical music (Xia, 2021).From the perspective of cognitivism education theory, it cannot adapt well to the existing knowledge structure of Chinese students, which creates a bottleneck for Chinese students in acquiring new knowledge. Therefore, a teaching method that attempts to solve this problem has been derived.In the abstract art language courses for painting majors in Chinese colleges and universities, the idea of combining Kandinsky's teaching method and Chinese calligraphy teaching method is generally adopted. However, traditional Chinese calligraphy teaching methods also have problems such as limitations of abstract interpretation and incoherent modules(Zhou, 2018).The new Chinese calligraphy teaching method I created is to solve the problems of traditional Chinese calligraphy teaching method in abstract art language courses.It is composed of three major modules. They are 111 training module, random design module, and instinct color module. The 111 training module focuses on the improvement of the teaching method of the abstract sketch module.
\end{abstract}

\section{Abstract art language courses and 111 training modules}

The abstract art language course is divided into two important modules, one is abstract sketching module, and the other is abstract color module (C. Wang, 2021).Figure 1 shows the relationship between the various modules of the course and the teaching methods in the traditional curriculum. 


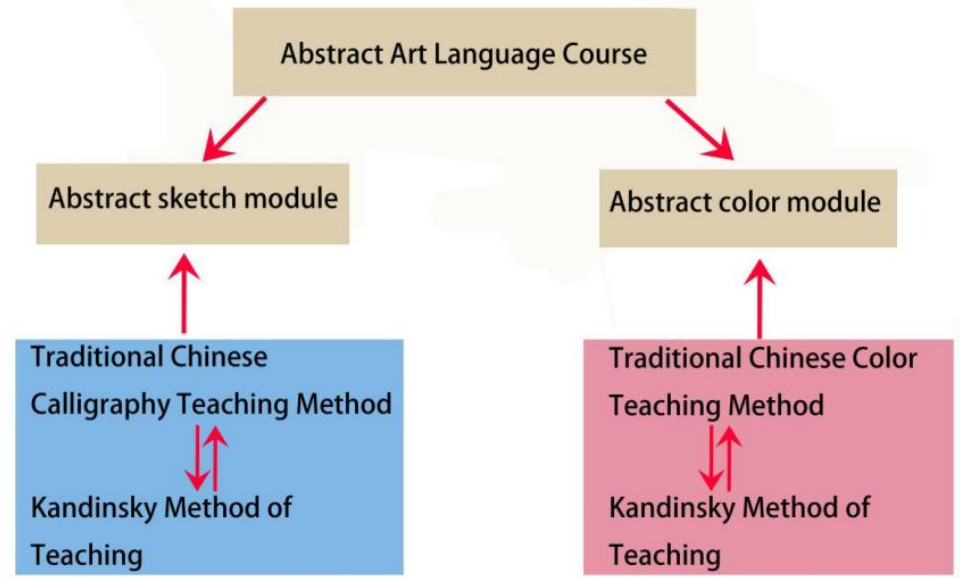

Figure 1.

Figure 2 shows the position of the 111 module in the new Chinese calligraphy teaching method. It replaces the traditional Chinese calligraphy teaching method and focuses on the improvement of the abstract sketch module.

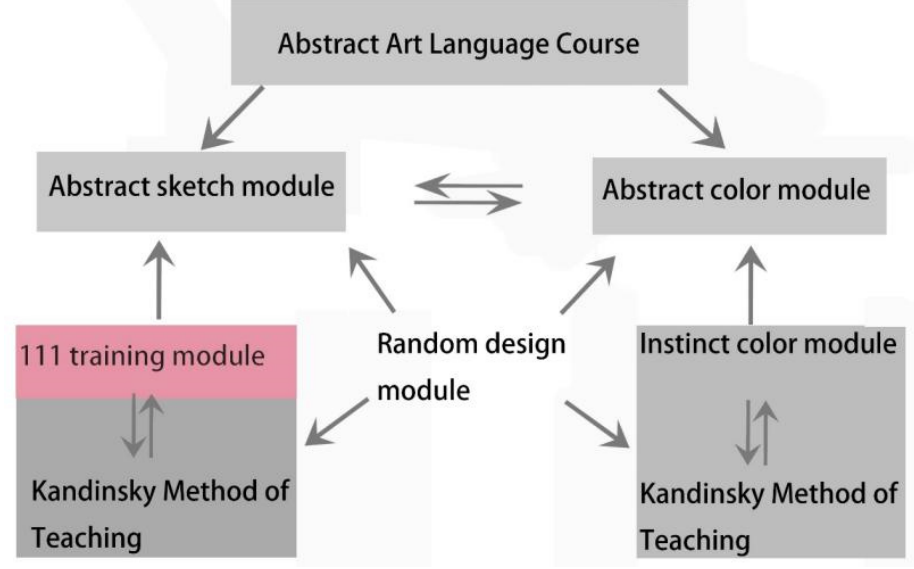

Figure 2.

In the new teaching method, the biggest difference between the 111 module and the traditional teaching method is:

First, The exercises in the new teaching method only choose one calligraphy style, one calligrapher, and one work for in-depth research. The exercises in the traditional teaching method are composed of multiple works of eight calligraphy styles by multiple calligraphers .

Second, the classroom exercises in the new teaching method focus on shape research and creative design. The practice of traditional teaching method focuses on copying Chinese calligraphy .

\section{Six exercises of 111 training module in new Chinese calligraphy teaching method}

The 111 training module chooses: Gu Shi Si Tie by Zhang Xu in the Kuang Cao style of Tang Dynasty in China.Figure 3 shows the full picture of Zhang Xu's Gu Shi Si Tie, which is now in the collection of the Liaoning Provincial Museum in China. It has 40 lines and 188 characters.

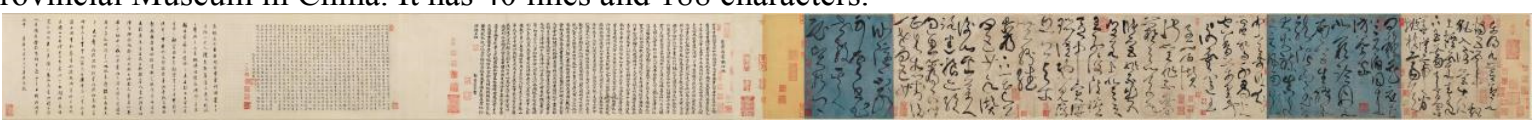

Figure 3. The complete picture of Zhang Xu's Gu Shi Si Tie

In the new teaching method, teachers can repeat the following 6 trainings according to the amount of class time. The exercises of the 111 training module are composed of these 6 parts, which correspond to the training of the following 6 abilities.

A.Space coloring in Zhang Xu's works (unconventional observation method)

Conventional observation method: Students will move their viewpoints by staring at the sequence of strokes of the characters. This affects students' attention to shape (Y. Wang, 2021). Although the literary meaning of Zhang Xu's works is not highly recognizable, it is a kind of text after all.The book General Specification for Stroke Order of Chinese Characters published by the National Language Working Committee of the Ministry of Education of the People's Republic of China in 2020. It stipulates the writing order of Chinese characters, which is the order that has been deeply imprinted in the minds of students since the elementary school level.Figure 4 shows the writing 
sequence of the Chinese character Yong. The number label represents which part to write first and which part to write later, and the arrow represents the trajectory of the pen.

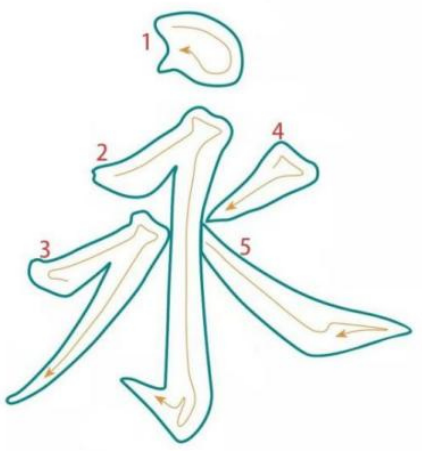

Figure 4.

Unconventional observation methods: The observation method of abstract painting art should have corresponding discipline requirements, and it should focus on shape research (S. Zhang, 2017).The purpose of this training is to exercise students' basic observation methods of shape. Its focus is to establish the shape awareness of students' planes.Avoid students moving their viewpoints in the order of strokes.In this training, students can use classic sketching methods to express. For example, students can use black, dark gray, light gray, and white to paint. They can also use different sketch lines to knit, or even choose any color to paint.It is worth noting that although students are allowed to use color in this training, coloring is only to facilitate the distinction between the shapes of different areas. Its research focuses on the difference in shape, which still belongs to the category of sketching research. Assignment A only pays attention to whether the graphics are found correctly, and does not discuss the theory of colors.

For the intuitiveness of the schematic diagram, this study uses the method of coloring for labeling. In training A, it is divided into 4 steps.First, the partition of Zhang Xu's work. Second, the coloring of closed shapes inside Zhang Xu's fonts. Third, the shape of the connection between characters of Zhang Xu's works is colored. Fourth, coloring the shape of row to row of Zhang Xu's works.

First, the partition of Zhang Xu's work.First, when students get the distributed material, they are required to carefully observe the material and divide the material into different levels. This step is very similar to the work of a director in casting roles, first a rough selection, and then a fine selection.Figure 5 is a part of Zhang Xu's Gu Shi Si Tie, where the thickest, deepest, and densest strokes should be the focus of the material. Then at least the things in this work can be roughly divided into two categories. The pink area represents the focus of the material, and the other areas represent the non-key areas.

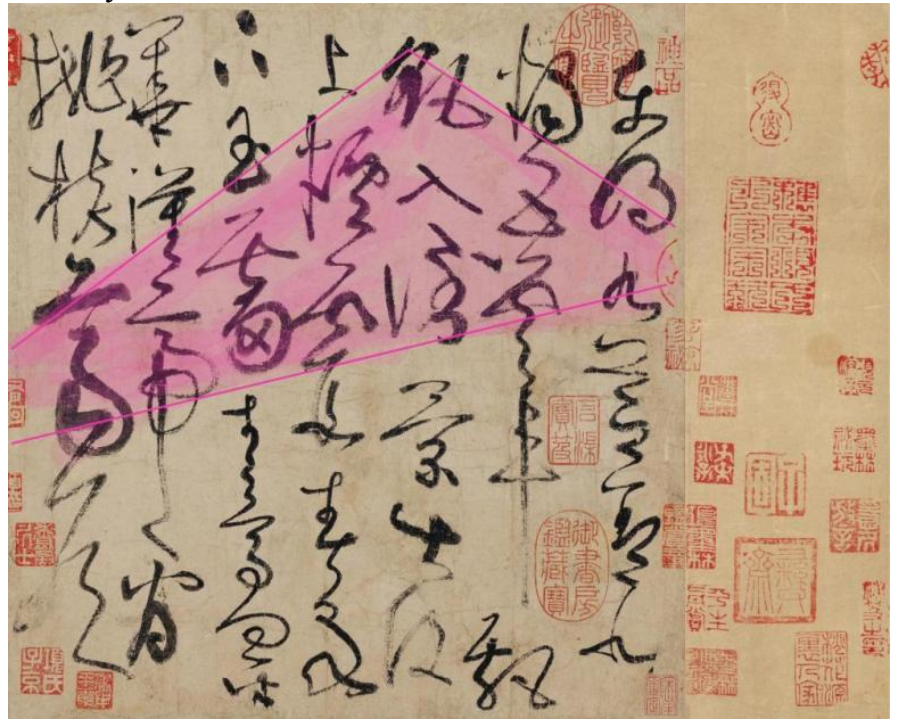

Figure 5. Schematic diagram of pink key areas

Then take a closer look at the more conspicuous elements in the non-key areas. For example, the green areas in Figure 6 are the more conspicuous elements in the non-key areas, because their shapes are darker and thicker or are significantly different from the surrounding shapes. 


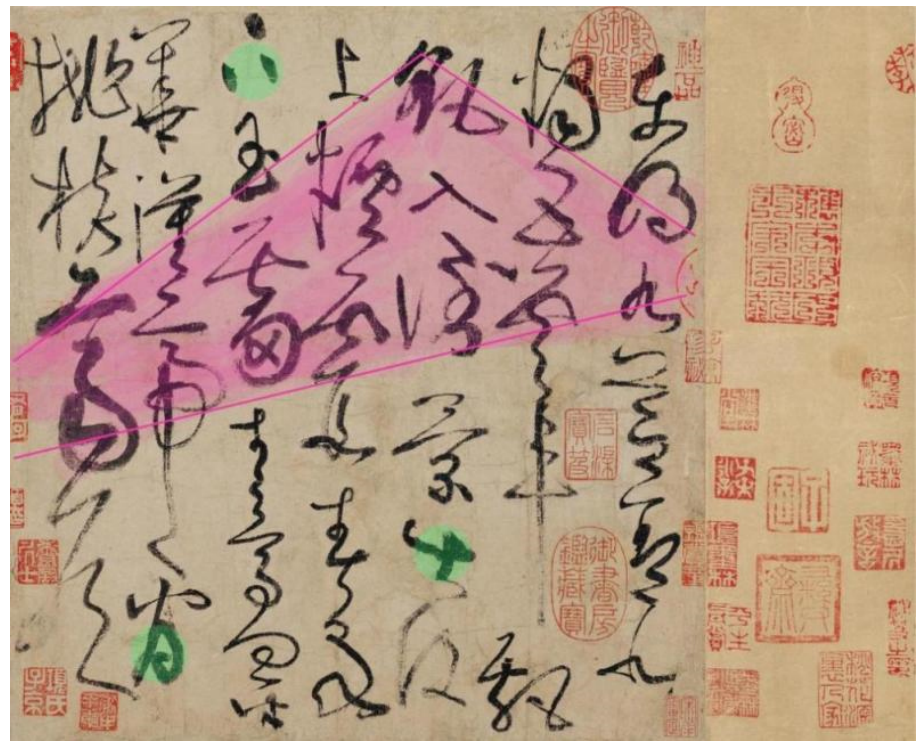

Figure 6. Schematic diagram of secondary extraction of non-key areas

In this way, the shape of the whole work is divided into three major categories. The first category is the pink focus area. The second category is the green area. The third category is the unpainted area.This step is to establish the overall awareness of the students' works. It is the deceptively simple and most important step. The three steps after this step are carried out on the basis of this one step.

Second, the coloring of closed shapes inside Zhang Xu's fonts. On the basis of the previous step, all the closed shapes of the work will also be divided into three categories. Students should express the different closed shapes in these three levels in three different ways.For example, students can choose three different colors for coloring, or use classic sketch drawing techniques for full coloring, cross-line coloring, and single-line coloring.Figure 7 is a schematic diagram of filling three different levels of closed shapes with red, blue, and green.

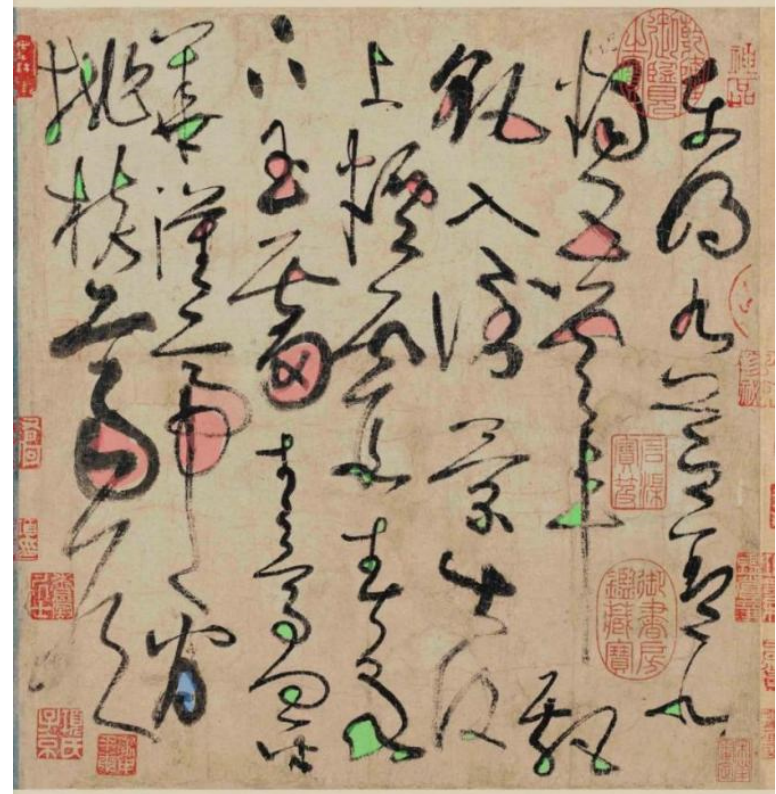

Figure 7. the coloring demonstration diagram of the closed figure formed by three different levels of fonts.

Third, the shape of the connection between characters of Zhang Xu's works is colored.The training focus of this step is to let students experience the shape relationship formed on the outside of the font.It is worth noting that in ancient Chinese calligraphy works, the order of reading is vertical reading and writing from right to left. Therefore, most of the works are vertically connected shapes. But it does not mean that students only need to pay attention to the longitudinal shape in this training. This is because the triangular pink key area in Figure 5 will not only have a vertical connection shape, but also a horizontal connection shape. The vertical connection shape is due to the writing and reading order of the text. The horizontal connection shape is because the triangle formed by the key area has a horizontal stretch. The non-key areas do not have the stretch of triangles, so there is no horizontal connection shape.Figure 8 uses green to represent the connection relationship between the vertical characters and the shape of the characters, and blue represents the connection relationship between the horizontal characters and 
the shape of the characters.

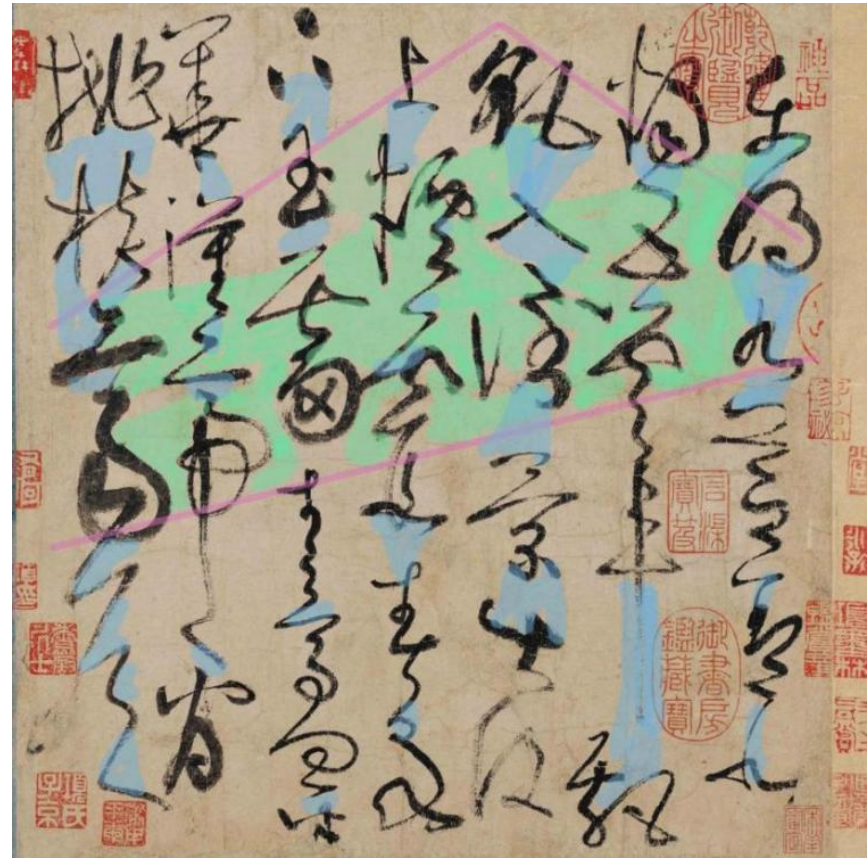

Figure 8. Example of coloring training for connecting shape of characters and characters

Fourth, coloring the shape of row and row of Zhang Xu's works. This training requires students to focus on the changes in the appearance of the row and row in Zhang Xu's works. At the same time, this training can make students pay attention to a larger area and further exercise their awareness of shape observation. Figure 9 uses green to represent the connection relationship between row and row shapes.

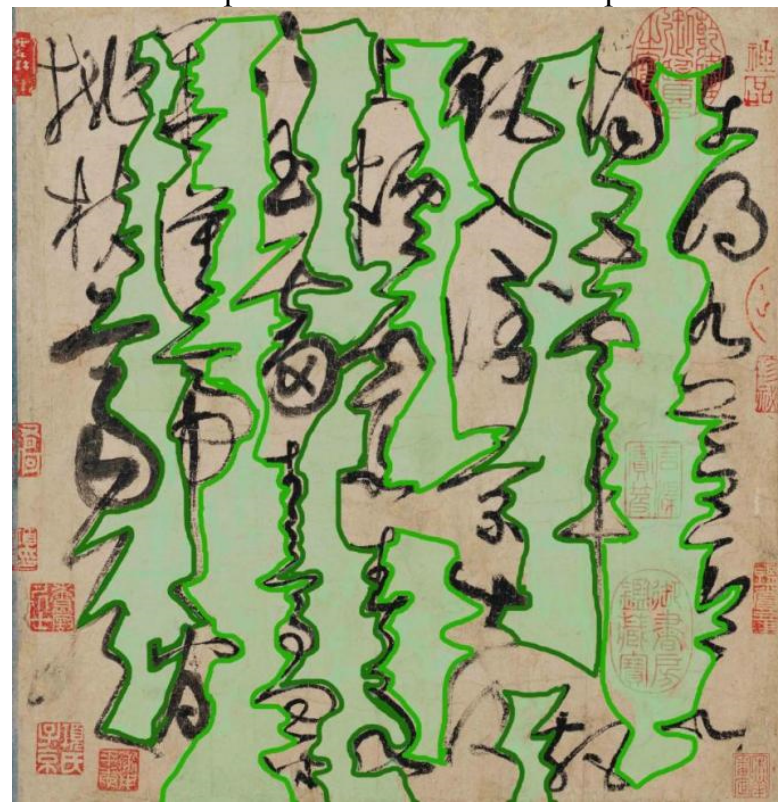

Figure 9. Example of coloring training for the connection shape of rows and rows

B. Pencil Sketches of various shapes of Zhang Xu's works (picking analysis of design elements)

On the basis of the previous training A, use a pencil to draw all the above shapes on the drawing paper. Further experience the exquisite design of Zhang Xu's works, and find the rules from it. Figure 10 is the design element picking analysis diagram made by student 01 in the experimental group. 


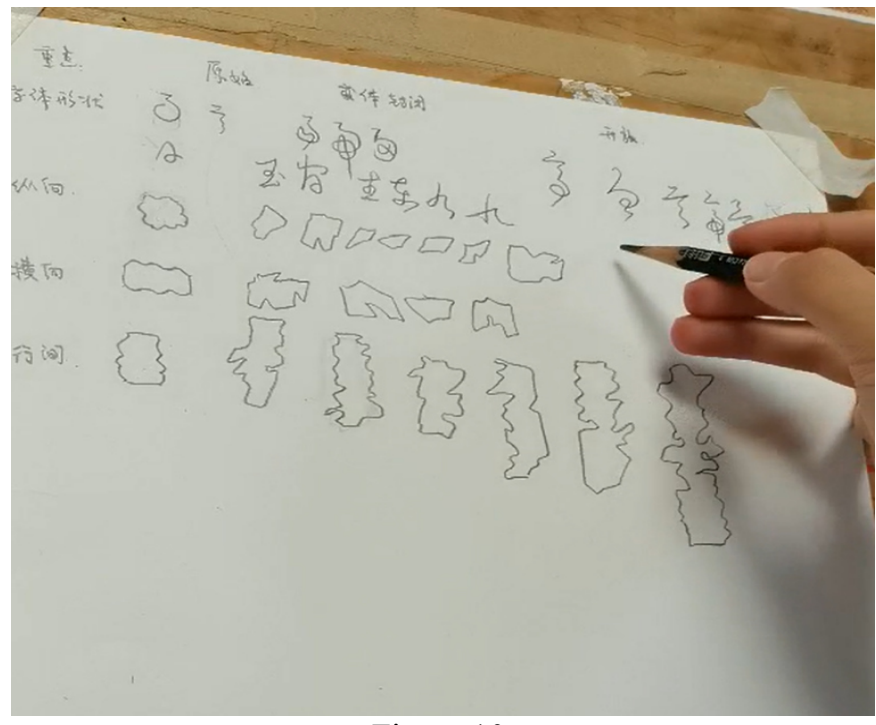

Figure 10.

C.Ink and wash sketches of various shapes in Zhang Xu's works (enhance drawing analysis for the characteristics of design elements)

On the basis of the previous assignment, use ink to trace the shape drawn on the drawing paper. When drawing this time, pay attention to the influence of line thickness, speed and other factors on the shape. Figure 11 shows the different visual effects of different lines on the same shape.
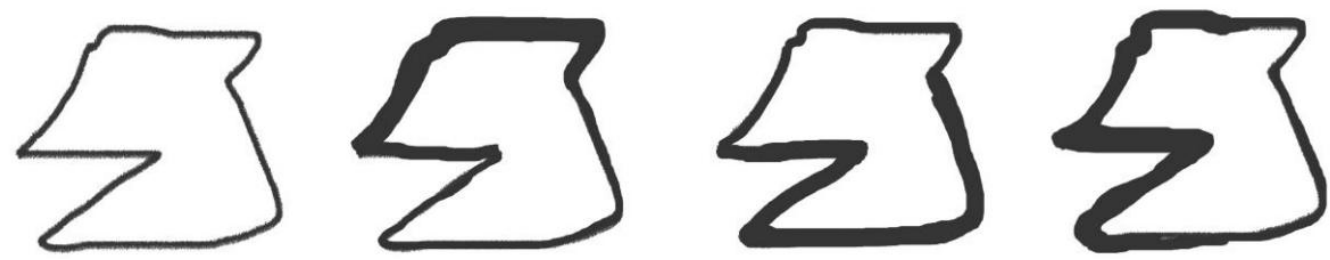

Figure 11

This assignment requires students to further appreciate the intricate design in Zhang Xu's work. At the same time, strengthen students' precise control over the brush and accurate grasp of the shape. Figure 12 is an example diagram of enhanced drawing and analysis of element characteristics of student 01 in the experimental group.

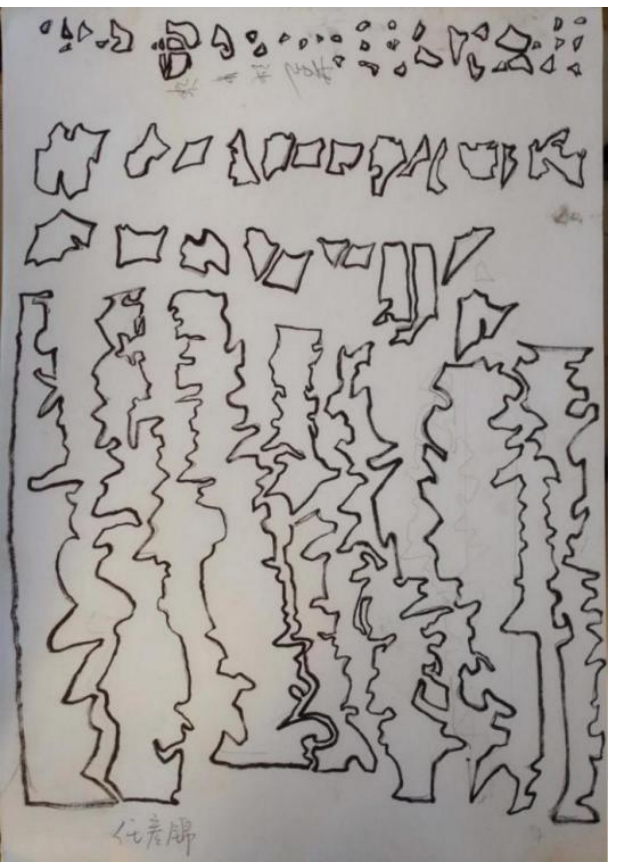

Figure 12.

Creative Copying of Zhang Xu's Works (Experience and Application of Abstract Lines)

This training is a long-term repeated training. It requires this training to be carried out at the beginning of each 
class. However, the copying in this training is not the copying in the traditional teaching method. The requirement of traditional copying is to be as consistent with the original as possible. Figure 13 is a work drawn by students in accordance with the requirements of traditional teaching copying.

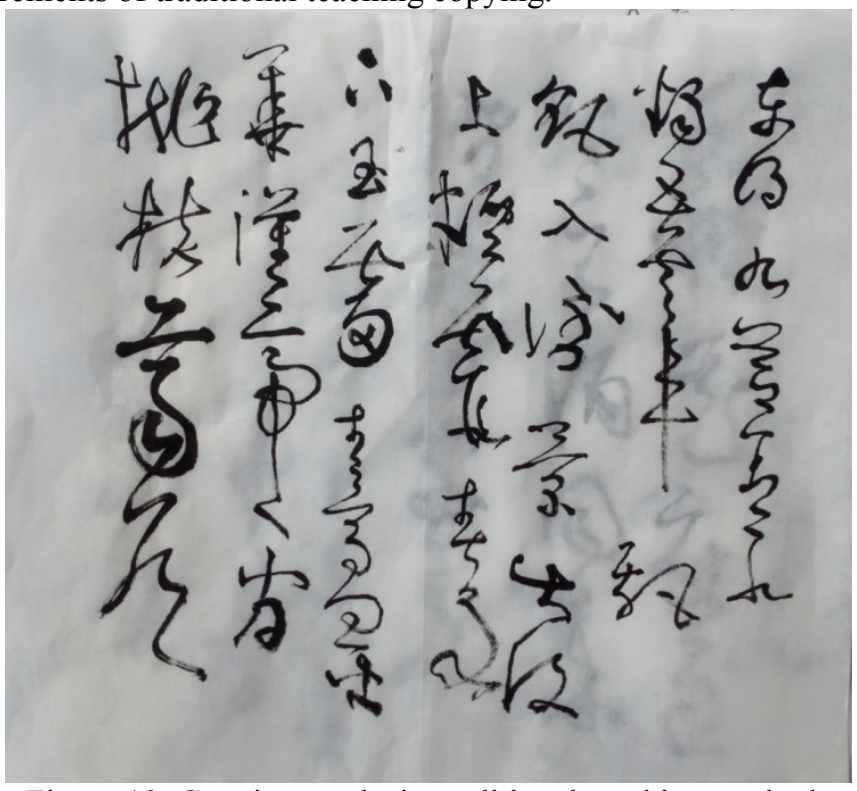

Figure 13. Copying works in traditional teaching methods

In the 111 training module, students are required to take the initiative to copy analysis and design a set of their own painting language system. Visually encode key fonts, non-key fonts, and special fonts. Figure 14 is a copy of the work of student 02 in the experimental group. She carried out hollow design, dotted line design, thickening design, and weakening design for the font. Developed its own visual coding design.

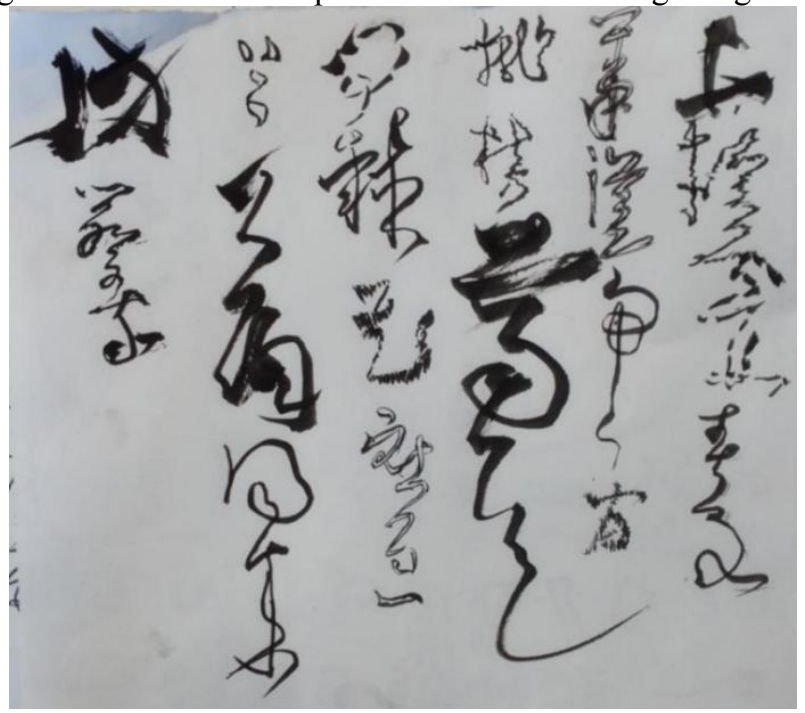

Figure 14. Copy works designed by students in the experimental group

E. Zhang Xu font analysis (crack the deformed password)

This training will send the interpretation of Zhang Xu's "Gu Shi Si Tie" to every student, with the purpose of letting every student understand Zhang Xu's design ideas for modifying standard Chinese fonts. The red font in Figure 15 is a standard Chinese font. It corresponds to Zhang Xu's deformed font in his works. 


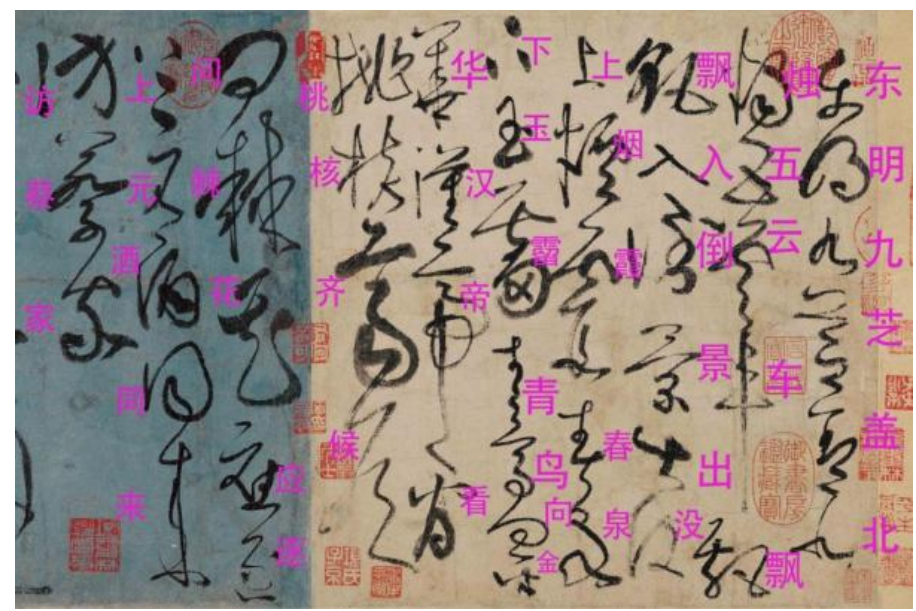

Figure 15. Comparison of standard Chinese fonts and Zhang Xu's works

The main purpose of this training is to make students understand which strokes Zhang $\mathrm{Xu}$ omitted in the font design, which strokes were added, and which strokes were modified. Students are required to perform three classification analyses on Zhang Xu's material. The first is a subtracted font. The second type is the font with addition. The third type is the font that has been replaced. Figure 16 is a font analysis diagram drawn by student 01 in the experimental group.

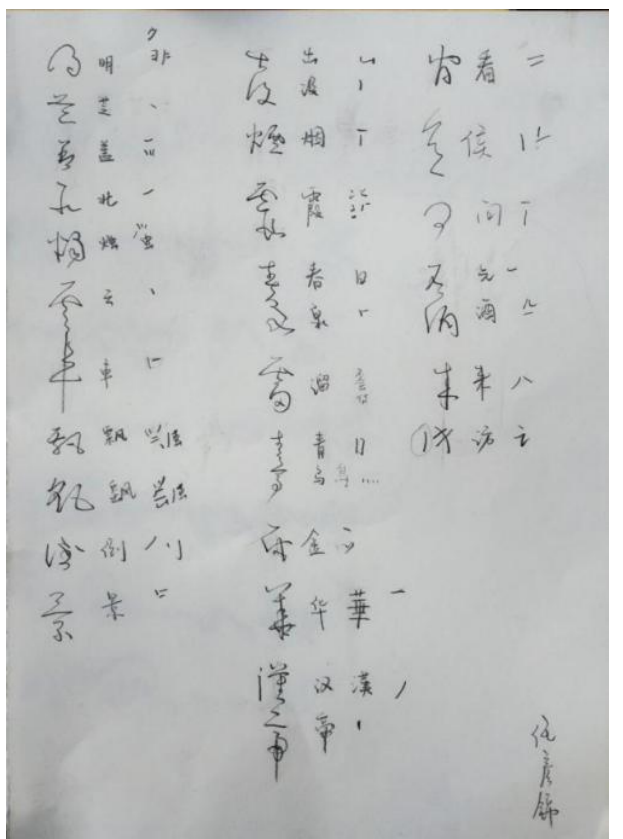

Figure 16. The font analysis diagram drawn by student 01 in the experimental group

This training can also make students understand that abstraction in Chinese calligraphy is not completely free. It is all evolved from an original standard Chinese font. After the analysis, students are required to select their favorite characters in Zhang Xu's works for secondary design. Students can further improve their design ability and understanding of Zhang Xu's works by comparing their own designs with Zhang Xu's works. This training does not focus on students' pen and ink skills, but focuses on students' analytical and design abilities.

F.Fill in the blanks of Zhang Xu's works (shape reasoning)

This training is an upgraded version of Zhang Xu's work analysis. Figure 17 is a picture of Zhang Xu's work sent to students. This training requires students to first find out the shapes of continuous variants in this work. This is to further allow students to deepen the impression of the previous exercises and improve their observation skills. 


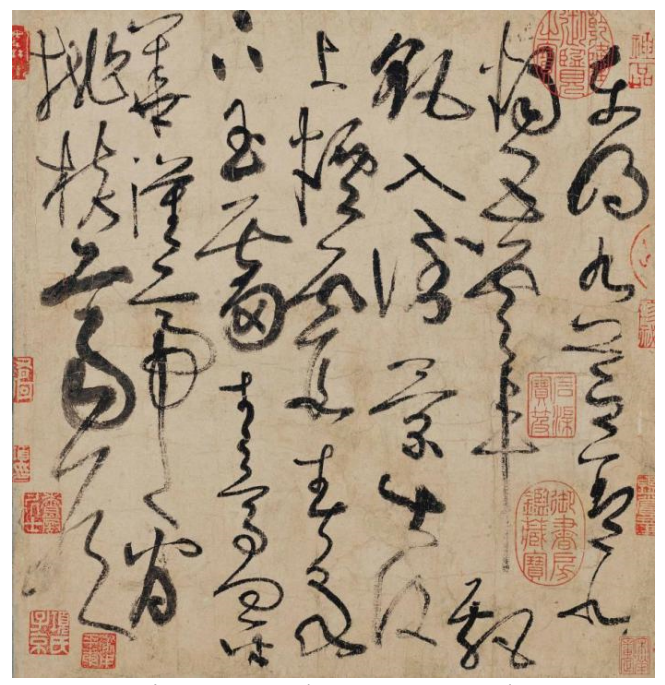

Figure 17. Zhang Xu's works

We extract a part of this work, taking the horizontal characters in the middle as an example. Fig. 18 is a character template of continuous variation in shape taken horizontally. In Figure 18, there are two basic elements that can be picked up. This is very similar to the English letters $\mathrm{Z}$ and $\mathrm{O}$ written vertically.
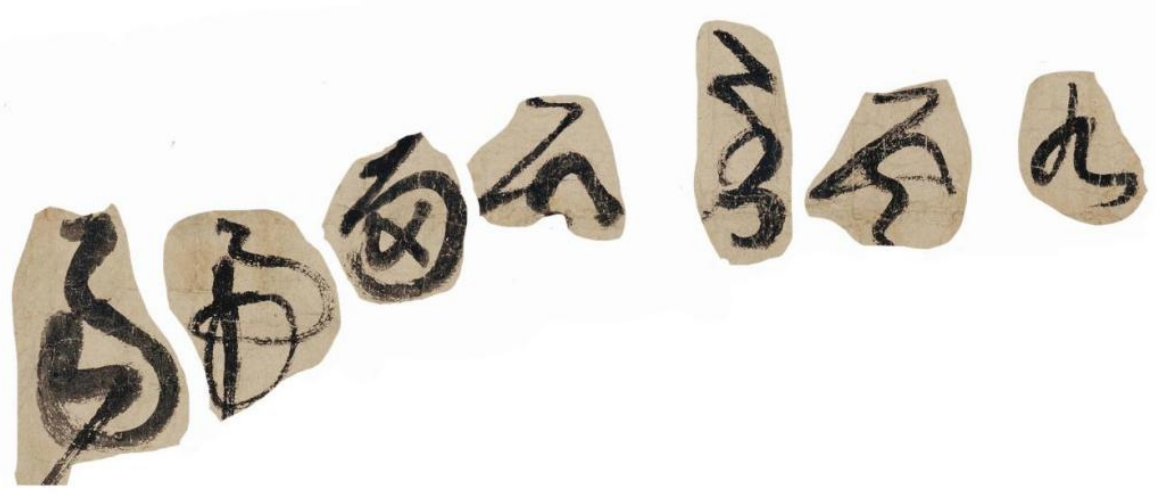

Figure 18. Schematic diagram of continuous variants of shapes taken in Zhang Xu's work.

Figure 19 is a schematic diagram of further analysis of Zhang Xu's work. Green represents "Z" and red represents "O". On this basis, students are required to draw a derivation diagram of the gradual change in shape.

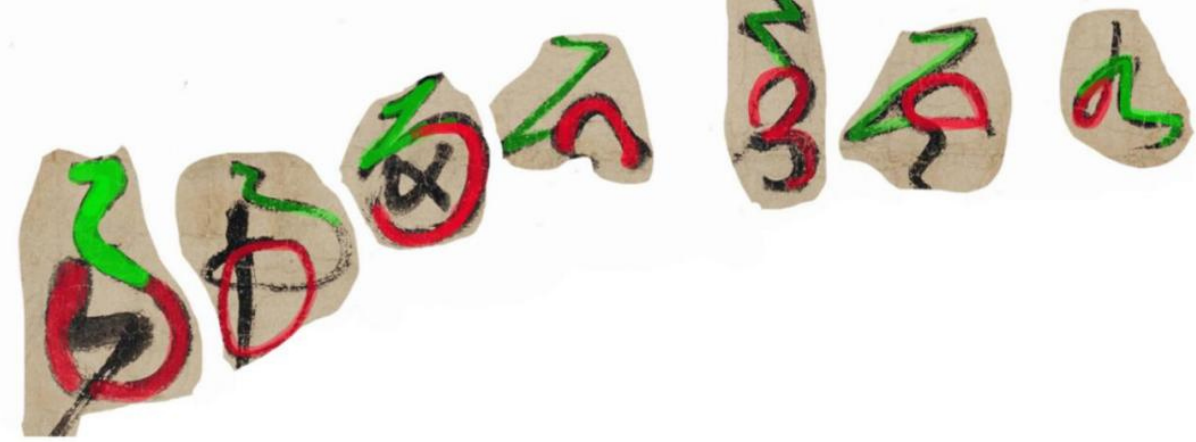

Figure 19. Schematic diagram of further analysis of Zhang Xu's works

Figure 20 is a shape derivation diagram drawn by students. We can see in this picture that the students summarized the shapes in Zhang Xu's works, and deduced the mother shape in Zhang Xu's works. This picture shows that Zhang Xu has designed "Z" and "O" 7 times. 


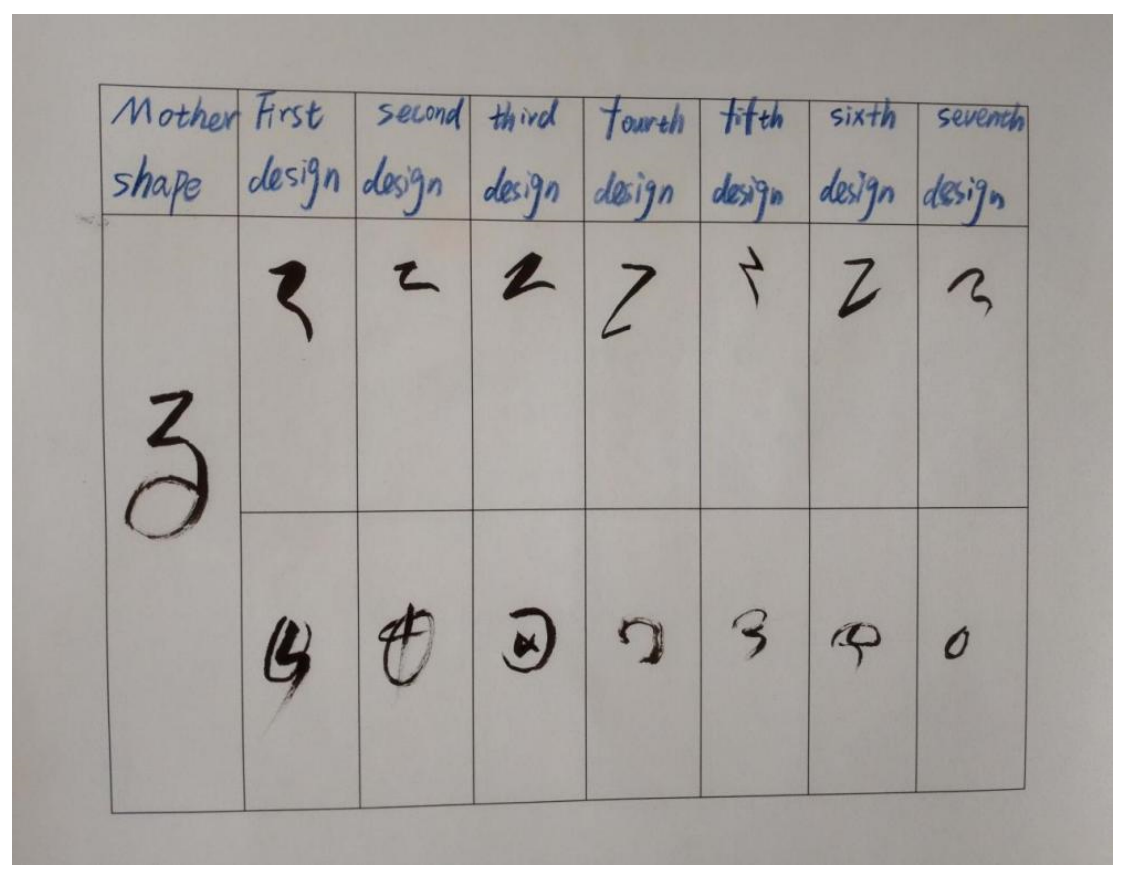

Figure 20. The shape derivation diagram of Zhang Xu's work drawn by students

On this basis, throw a question to the students: if you were $\mathrm{Zhang} \mathrm{Xu}$, how would you design? The new teaching method requires students to fill in the blanks design to inspire students' design thinking. Figure 21 is a schematic diagram designed by students.

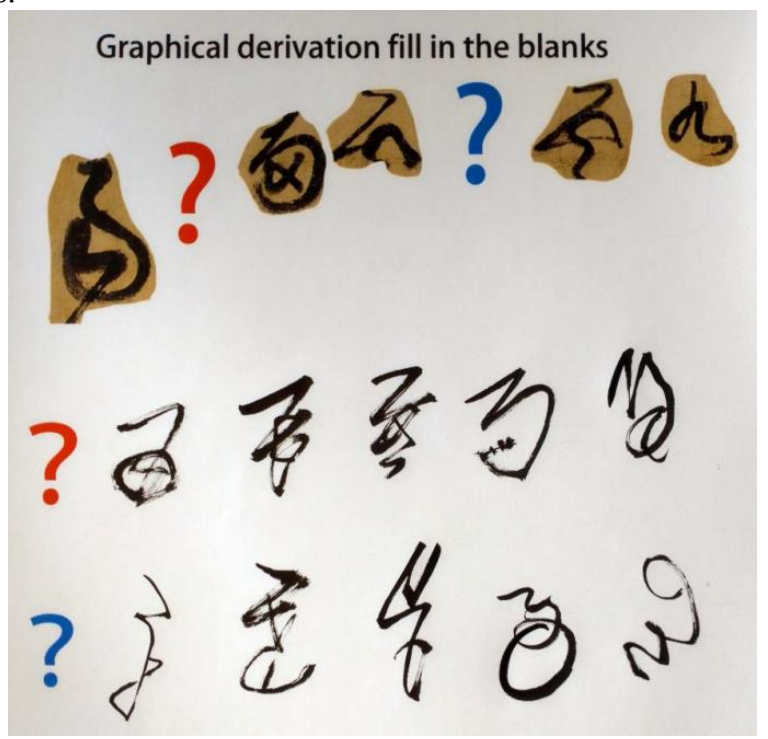

Figure 21. Five designs of students' fill-in-the-blank fonts

This training question is more difficult than the previous training question. It requires students to find continuous variants of related shapes in Zhang Xu's works, and to do fill-in-the-blank designs. This is done to stimulate students' logical reasoning ability about shapes and to stimulate students' awareness of active design.

\section{Conclusion}

The six exercises involved in this study follow Bruner's learning theory. Bruner's learning theory emphasizes the importance of knowledge structure and that learning is an active process (Yuan, 2021). The first step in the painting process is observation. The second step is to think. The third step is to pick up the brush and paint. The fourth step is to rethink. The cycle goes on and on (M. Zhang, 2021). Figure 22 is a schematic diagram of the relationship between the drawing learning structure and each training. 


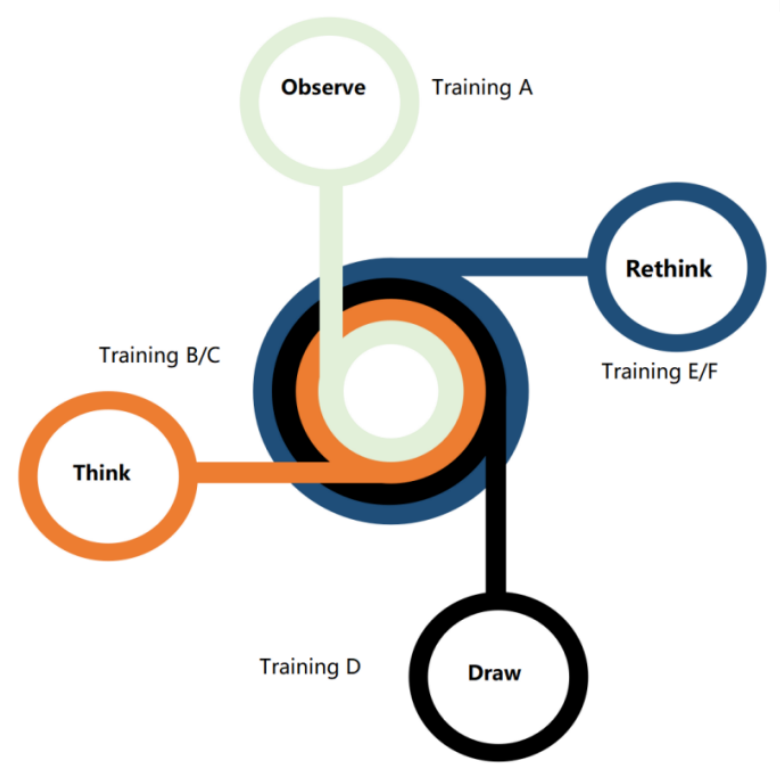

Figure 22. Schematic diagram of the relationship between the drawing learning process and each training

The six trainings in this study are from shallow to deep. The content of each training is gradually advanced on the basis of the previous training. First, start with the observation method, and train A to cultivate the observation method of students' abstract art. Training B is to generalize the observed phenomena. Training $\mathrm{C}$ is a reanalysis of the inductive summary. Training $\mathrm{D}$ is to solve the problem of how to pick up a brush and paint. Training $\mathrm{E}$ is to reflect on previous design work. Training $\mathrm{F}$ is to re-search for materials and redesign after reflection.

\section{References}

Luo, Y. (2013). A Comparative Study of Chinese and Western Abstract Paintings. Popular Literature and Art, $328(22), 126-127$.

Wang, C. (2021). Abstract art language. Southwest Normal University Press.

Wang, Y. (2021). The exploration of cursive script teaching under the background of "poetry education"

Taking "Gu Shi Si Tie" and "Xiao Jing" as examples. Jiangsu Education, 65(12), 32-39.

Xia, Q. (2021). Vasily Kandinsky: The Road to Abstraction. Art China, 20(04), 22-25.

Yuan, B. (2021). The Enlightenment of Bruner's Cognitive Theory to the Reform of Management Basic Courses in Higher Vocational Colleges. Future and Development, 45(10), 91-94.

Zhang, M. (2021). Analysis of Characteristics and Methods of Aesthetic Appreciation in Chinese Painting Theory. Journal of Nanjing Arts Institute(Fine Arts \& Design), 43(05), 146-151.

Zhang, S. (2017). Research on the Teaching System of Abstract Painting. Writers and Artists of China, 103(10), 174.

Zhou, X. (2018). On the Selection of the Introductory Style of Calligraphy for College Students Majoring in Fine Arts. Journal of Sichuan Minzu College, 27(06), 74-76. 